\section{Choice of neuroleptics in epilepsy}

Sir: McConnell et al provide a first-rate overview of the difficulties associated with the use of neuroleptics in patients with comorbid seizure disorders (Psychiatric Bulletin, October 1997, 21. 642-645). However, we feel that the difficulties associated with clozapine use in such patients are often overemphasised. Although clozapine is known to lower the seizure threshold and commonly produces non-specific electrocardiogram changes, clinical data would suggest that seizures are rarely an insurmountable obstacle to its use.

While pre-marketing studies did find clozapine to be associated with a relatively high incidence of seizures, post-marketing surveillance by the Clozaril Patient Monitoring Service (CPMS) does not appear to confirm this. Data collected by the CPMS on 99502 patients between 1988 and 1994 found a seizure rate of $1.3 \%$ in the USA (Pacia \& Devinsky, 1994; Honigfeld, 1996). Of those patients who did have seizures, half were concurrently receiving other medications known to lower the seizure threshold and a third had prior history of seizures. Only $0.4 \%$ of all patients had recurrent seizures, the others were isolated single events during initial dose titration, and three-quarters of these patients were able to continue taking clozapine with more gentle dose titration or the addition of an anti-convulsant. Thus, only one in a thousand patients had to discontinue clozapine therapy due to intractable seizures.

Since the prevalence of epilepsy in the general population is thought to be $3-4 \%$, the above data allow us to estimate that fewer than $10 \%$ of patients with a history of epilepsy had even a single seizure while taking clozapine and, at most, $3 \%$ of patients with epilepsy had to discontinue clozapine therapy due to an unmanageable exacerbation of their pre-existing seizure disorder. European studies have found even lower seizure rates (Naber et al, 1992).

The use of clozapine does require special precautions and increased vigilance, particularly in patients with seizure disorders, and it would certainly not be a first-choice neuroleptic in such patients. However, it would be unfortunate if patients suffering the devastating consequences of treatment-resistant schizophrenia were denied the unique benefits of clozapine because of excessive concern about its possible adverse effects.

HoNigfeld, G. (1996) Effects of the clozapine national registry system on the incidence of deaths related to agranulocytosis. Psychiatric Services, 47, 52-56.

Naber, D., HolzBaCh. R., PerRo, C., et al (1992) Clinical management of clozapine patients in relation to efficacy and side-effects. British Journal of Psychiatry, 160 (suppl 17), 54-59.
Pacia, S. V. \& DeVINSKY, O. (1994) Clozapine-related seizures. Neurology. 44, 2247-2249.

JAMES G. LONGHURST and ERICA L. WEISS, Abraham Ribicoff Research Facilities, Department of Psychiatry and Connecticut Mental Health Center, Yale University, 34 Park Street, New Haven, Connecticut 06519

\section{Care Programme Approach}

Sir: As the Care Programme Approach (CPA) dictates a particular form of doctor-patient relationship it is an active treatment technique rather than a neutral administrative tool. As such it should be practised within the bounds of experience, research and common sense.

Good assessment, discussing treatment options with the patient, communicating with involved colleagues and an interest in follow up are good practice. One of the most essential cornerstones of the assessment process is the decision as to whether or not the patient can and should take responsibility for their own behaviour, including their health-seeking behaviour. For most patients we assess them as able to hold that responsibility. This does not mean that these patients are less ill, less disabled or less in need of well-resourced treatment plans than those on the highest level of CPA or indeed that they are less capable of suicide and/or murder than the general population. For many patients it is important to remind them that they do retain responsibility for their actions in case in the heat of their distress or the disorder of their personality they have forgotten.

CPA as presently designed is too blunt a tool. too uni-axial and too all inclusive to be helpful. A small group of patients can benefit from CPA but there is a danger that psychiatrists will breed hostility to it because it is an irrelevant process for the majority of their patients. For some patients, particularly those with personality problems, CPA is contraindicated. Hearing even the slightest hint that someone else may be held responsible for their behaviour would be enough to destroy any hopes of a therapeutic relationship. CPA also seems at odds with the ethos of many of the psychological therapies.

Rather than universal CPA we need reasonable resources: with those we can serve the best needs of all our patients.

L. JANE KNOWLES, 7 Albert Road, Caversham, Reading RG4 7AN and REX HAIGH, Highbury, 1 St Mary's Road, Mortimer RG7 3UE

\section{Unfitness to plead in Scotland}

Sir: The procedure for dealing with cases of 'insanity' in Scotland was changed fundamentally 